\title{
Werden die Neubaugebiete der 1960er- und 1970er-Jahre zu Problemgebieten?
}

\author{
Andreas Klee ${ }^{1}$ \\ Online publiziert: 23. Januar 2018 \\ ๑) Springer-Verlag GmbH Deutschland, ein Teil von Springer Nature 2018
}

Die 1960er- und 1970er-Jahre waren von intensiven Neubautätigkeiten geprägt, gerade auch im Segment der Einund Zweifamilienhäuser. In der Zeit wuchsen die Vorstädte erheblich, aber auch in vielen ländlichen Räumen entstanden diese Gebäudetypen in großem Umfang. Sie entsprachen dem damaligen Bild von zeitgemäßem Wohnen: im Grünen, mit hohem Komfort und viel Platz für die Familie. Vor allem ging der Bau der Ein- und Zweifamilienhäuser mit einer schnell steigenden Autoverfügbarkeit der Haushalte einher. Daher entstanden viele Siedlungen fernab des schienengebundenen Nahverkehrs und waren oftmals gar nicht oder nur umständlich mit Bussen zu erreichen. Das nahm man seinerzeit jedoch ohne Weiteres in Kauf.

Inzwischen sind die seinerzeitigen Erbauer der Ein- und Zweifamilienhäuser in ein Alter gekommen, in dem plötzlich die Nachteile dieser Wohnform sichtbar werden. Das Haus ist nach Auszug der Kinder zu groß, die Gartenarbeit zu beschwerlich und der fehlende öffentliche Verkehr wird zum Problem beim täglichen Einkauf oder beim Arztbesuch. Häufig finden sich auch keine Nachmieter oder Käufer für die ,in die Jahre gekommenen“ Immobilien. Werden nun diese früheren Neubaugebiete zu Problemgebieten? Die Antwort ist nicht einfach. Denn einzelne Regionen sind von dieser Entwicklung in unterschiedlichem Maß betroffen. Darauf gehen Brigitte Adam, Andrea Berndgen-Kaiser, Kerstin Jochimsen, Angelika Münter und Philipp Zakrzew$s k i$ in ihrem Beitrag ausführlich ein. Sie entwickeln am Beispiel von Nordrhein-Westfalen ein Indikatorenkonzept zur Abschätzung der Situation der Ein- und Zweifamilienhausbestände der 1950er- bis 1970er-Jahre und zeigen, dass erhebliche Unterschiede zwischen peripheren Regionen und prosperierenden Städten, somit unterschiedliche Marktsituationen und Entwicklungsperspektiven für dieses Woh-

Prof. Dr. Andreas Klee

klee@arl-net.de

1 Akademie für Raumforschung und Landesplanung, Leibniz-Forum für Raumwissenschaften, Hohenzollernstraße 11, 30161 Hannover, Deutschland nungsmarktsegment bestehen. Daraus resultieren auch sehr unterschiedliche Notwendigkeiten des kommunalen Handelns.

Die Entwicklung des Marktes für Ein- und Zweifamilienhäuser hängt zu einem beträchtlichen Teil auch mit altersbedingtem Wohnungswechsel zusammen, denn oft wird ein Haus von den Bewohnern aus Altersgründen aufgegeben. Der Beitrag von Uwe Engfer befasst sich mit den aktuellen Tendenzen der Binnenmigration älterer Menschen und geht dabei der Frage nach, ob und inwieweit ein Trendwechsel in den Herkunfts- und Zielgebieten der Senioren hin $\mathrm{zu}$ einer Reurbanisierung stattfindet. Während in der Vergangenheit Binnenwanderungen im Alter mehrheitlich aus urbanen Räumen in die Peripherie oder in ländliche Regionen erfolgten, wird derzeit häufig von einer gegenläufigen Tendenz berichtet. Uwe Engfer kommt zu dem Ergebnis, dass es einen „bemerkenswerten Strukturwandel der Ruhestandsmigration" gibt, denn immer mehr Großstädte weisen positive Migrationssalden auf, insbesondere in Ostdeutschland. In der Mehrzahl der Großstädte im Westen ist jedoch das alte Muster noch vorherrschend, aber es lässt sich ein deutlicher Rückgang der Wanderungsverluste der Kernstädte feststellen. Außerdem wird ein genereller Rückgang der Wanderungen der Senioren festgestellt. Damit, so Engfer, seien die in den Ruhestand nachrückenden Kohorten zunehmend immobiler als ihre Vorgänger. Dies wird als Beleg dafür angesehen, dass sich die Bedingungen des Alterns am Ort in jüngster Vergangenheit erheblich verbessert hätten. Es zeigt sich, dass die Bestände an Ein- und Zweifamilienhäusern nicht von vornherein ihre Bedeutung als Wohnstandort im Alter verlieren.

Was bringen die weiteren Beiträge dieses Heftes? Thomas Meister thematisiert den „Ausbau von Offshore-Windparks in Deutschland aus einer Innovationsperspektive“. Der Autor stellt die Hypothese auf, dass die in der Anfangsphase des Ausbaus der Windparks implementierten Steuerungsinstrumente eine zügige Entwicklung blockierten. Durch die fehlende räumliche Steuerung seien Nutzungs- und Naturschutzkonflikte befördert worden und es sei ein „Wildwuchs“ der Offshore-Windparks entstan- 
den, der einer effizienten Netzanbindung entgegenstand. Die diesbezüglichen Steuerungsversuche der öffentlichen Hand untersucht Meister aus einer Innovationsperspektive mithilfe der Konstellationsanalyse.

Der Beitrag von Michael Fritsch, Martin Obschonka, Michael Wyrwich, Samuel D. Gosling, Peter J. Rentfrow und Jeff Potter präsentiert eine räumlich differenzierte Darstellung von unternehmerischen Persönlichkeitsmerkmalen in der Bevölkerung. Dieses Ergebnis der Kombination psychologischer (auf Basis des Big-Five-Modells) und geographischer Kompetenz ist innovativ und bearbeitet eine Forschungslücke, indem die ungleiche Verteilung individueller Merkmale zur Erklärung regionaler Entwicklung - genauer: regionaler Mentalitäten und entsprechender wirtschaftlicher Folgen - beitragen kann.
Schließlich widmen sich Paulina Schiappacasse und Bernhard Müller dem derzeit viel diskutierten Begriff der Resilienz. Der Beitrag basiert auf einer umfangreichen Analyse von Zeitschriftenaufsätzen in den Raum- und Umweltwissenschaften. Die Autoren untersuchen, wie Begriff und Konzept von Resilienz verwendet werden. und mit welchen Zielsetzungen, Deutungen, Wertungen und Desiderata Resilienz verbunden ist. Eine der Schlussfolgerungen der Autoren lautet, dass die Diskussion um Resilienzkonzepte künftig spezifischer geführt werden sollte. Sie brauche vermehrt transdisziplinäre Ansätze und eine stärkere regionale Kontextualisierung, insbesondere bei Studien zur Stadtund Regionalentwicklung in den Ländern des Globalen Südens.

Wir wünschen allen Leserinnen und Lesern eine anregende Lektüre. 